\title{
1 Raman study of lysozyme amyloid fibrils suspended on super-hydrophobic surfaces by shear flow
}

2 Manola Moretti ${ }^{1}$, Marco Allione ${ }^{1}$, Monica Marini ${ }^{1}$, Bruno Torre ${ }^{1}$, Andrea Giugni ${ }^{1}$, Tania Limongi ${ }^{1}$,

3 Gobind Das ${ }^{1}$ and Enzo Di Fabrizio ${ }^{1,2}$

$4 \quad{ }^{1}$ Physical Sciences and Engineering (PSE) and Biological and Environment Science and Engineering

5 Divisions (BESE), King Abdullah University of Science and Technology, Thuwal 23955-6900, Kingdom

6 of Saudi Arabia.

$7 \quad 2$ Bio-Nanotechnology and Engineering for Medicine (BIONEM), Department of Experimental and

8 Clinical Medicine, University of Magna Graecia Viale Europa, Germaneto, Catanzaro 88100, Italy.

9 Corresponding author: enzo.difabrizio@kaust.edu.sa

10 Abstract

11 The shear flow generated at the rim of a drop evaporating on a micro-fabricated super-hydrophobic

12 surface has been used to suspend and orient single/few lysozyme amyloid fibrils between two pillars for

13 substrate-free characterization. Micro Raman spectroscopy performed on extended fibers evidenced a

14 shift of the Amide I band main peak to the value attributed to $\beta$-sheet secondary structure, characteristic

15 of the amyloid fibers. In addition, given the orientation sensitivity of the anisotropic molecule, the Raman

16 signal of the main secondary structure was nicely enhanced for a fiber alignment parallel to the

17 polarization direction of the laser. The substrate-free sample generated by this suspending technique is

18 suitable for other structural analysis methods, where fiber crystals are investigated. It could be further

19 employed for generation of arrays and patterns in a controllable fashion, where bio-compatible material is 20 needed. 
21 Keywords: super-hydrophobic micro-patterned surfaces; Raman spectroscopy; amyloid fibrils; $\beta$-sheet 22 secondary structure

Introduction

Micro-patterned super-hydrophobic surfaces (SHS) are an ideal platform for the manipulation and characterization of single biological molecules and cells in background free environment [1-5]. The key element for suspending long molecules between two adjacent pillars is the shear flow generated at the rim of the evaporating drop [6]. This allows the single molecules to extend across the pillars top, ready for analysis. For example, literature reports DNA and DNA-protein complexes stretching over the micropillars substrates using this method, providing suitable samples to be analyzed by different techniques such as scanning electron microscope (SEM), transmission electron microscope (TEM) and Raman Spectroscopy [7-10]. In essence, when a drop of the solution containing the molecules of interest is

32 deposited over this kind of super-hydrophobic surface, it will shrink until a residue accumulates at its center. Through the process, suitably long molecules might pin at the edge of the pillars, and due to the evaporation, they are pulled until they bridge the adjacent pillars in the direction of the flow. This is possible because the shape and design of the micro-fabricated surface maintain the drop quasi-spherical while shrinking, favoring the hopping of the receding meniscus from one pillar's edge to the next. Importantly, by structuring the surface it is possible to spatially organize the stretched molecules.

The three dimensional structure of a functional protein has a central role for its activity in the cellular environment and is characterized by the co-presence of three main types of secondary structure, namely $\alpha$-helix, random coil, and $\beta$-sheet. Several pathologies are associated with the disarrangement of

41 the tertiary structure of the protein, for example the diseases related to amyloidosis [11]. Amyloid 42 deposits are characterized by the presence of highly oriented fibers made-up of $\beta$-strands organized into $43 \beta$-sheets and further into $\beta$-helix or solenoids, generated from the aggregation of misfolded or unfolded 
proteins which escaped the cellular quality-control [12]. The presence of $\beta$-sheet secondary structure can be confirmed by micro Raman spectroscopy, which is a non-destructive, label free technique especially suited for the localized biochemical analysis of biological samples. Appreciably, the results of Raman analysis can complement structural data obtained with other techniques such as NMR and x-ray spectroscopy, their most significant limitation being size $(<40 \mathrm{kDa})$ and the need for single crystals, respectively [13].

In Raman spectroscopy, the Amide I band position, largely shifting around $1650 \mathrm{~cm}^{-1}$, is routinely used to assign the $\alpha$-helix, random coil, or $\beta$-sheet composition of the proteins. The recent literature agrees in assigning to the $\beta$-sheet secondary structure a Raman shift in the range $1665-1675 \mathrm{~cm}^{-1}[14,15]$. An upshift of the Amide I band is evidenced in the transition from functional protein to amyloid structure, where $\alpha$-synuclein and insulin were considered [16, 17]. In 2007, a first report on functional amyloids

55 derived from different types of silk fibers, highlighted the potential of polarized Raman spectroscopy, 56 while assigning to the $\beta$-sheet secondary structure the Raman shift value in the range of $1666-1670 \mathrm{~cm}^{-1}$ 57 [18]. They observed that the spectra were dominated by the contribution of the $\beta$-sheet, and used the peak height of the polarized Amide I band collected in different scattering geometries to demonstrate that the main Raman tensor axis of the Amide I is highly oriented perpendicular to the fiber direction. A more recent polarized Raman spectroscopy study takes into consideration insulin amyloid fibers aligned on a

61 planar substrate by the coffee ring stain effect [19, 20]. There, an increased intensity of the Raman peak 62 centered at $1672-4 \mathrm{~cm}^{-1}$ emerges in the Raman spectrum when the fiber is aligned parallel to the laser 63 polarization, an indication of the cross- $\beta$ structure of the fiber core. the hen egg white lysozyme (HEWL) after its conversion to amyloid fibrils. The samples were prepared suspended across the gaps between neighboring pillars, and thus in a substrate and debris free 
intensity of the peak assigned to the $\beta$-sheet secondary structure to its orientation with respect to the exciting laser polarization, with a three-fold counts increase when oriented parallel to the laser polarization as compared to the perpendicular case. These results remark the ability of this technique to align single molecules along one given direction. Fiber suspension by shear flow on super-hydrophobic surfaces provides a fast and reproducible mean for the characterization of single molecules, purified form the solution buffer and debris, which typically represent a serious problem in standard preparation for laboratory analysis. The coupling of this preparation technique with Raman spectroscopy for the analysis of the single suspended fiber is then a powerful combination for the study of any anisotropic fiber crystal, where the structural characterization is not trivial by means of, e.g., x-ray crystallography or NMR.

Materials and Methods

\section{Super-hydrophobic surface fabrication}

Super-hydrophobicity emerges as a result of the patterning and functionalization of the surface, whose combination provides very high contact angle displayed by these substrates $\left(>150^{\circ}\right)$. In this case,

81 the pattern is a sequence of concentric circular arrays of pillars with radial pitch of $18 \mu \mathrm{m}$, each of the 82 pillars having a diameter of $6 \mu \mathrm{m}$ and height of 7 to $10 \mu \mathrm{m}$. To maintain a uniform surface coverage, the number of pillars in each circular array was increased according to the recursive formula $N_{i}=N_{i-1}+4$, where $N_{i}$ is the number of pillars of the $i$ th circle. The patterning of the surface was obtained with a combination of optical lithography and deep reactive ion etching (DRIE) technique. The original substrate was a 4" standard $\mathrm{Si}<100>$ wafer. In the first step, the pillars pattern was defined by means of negative optical lithography. The sample was then etched by means of DRIE (PlasmaLab System 100, Oxford Instr.), with a final height of the pillars of about $10 \mu \mathrm{m}$. Finally, the functionalization of the surface with hydrophobic material was achieved by the deposition of Perfluorodecyltrichlorosilane (FDTS) in a 
91 with $\mathrm{OH}$ groups which are exposed at the surface of the oxidized $\mathrm{Si}$, creating a monolayer of hydrophobic

92 fluoro-carbon chain molecules bound to the sample surface. The functionalizing FDTS was dispensed

93 together with water vapor in a vacuum pumped chamber of an MVD commercial system (MVD100E,

94 Applied MST).

\section{Lysozyme fibrils preparation}

Lysozyme amyloid fibrils were produced starting from a solution of HEWL powder (Sigma) [10 $\mathrm{mg} / \mathrm{ml}$ ] in MilliQ water based on [21]. Acidification of the solution to $\mathrm{pH} \sim 2.0$ was obtained by adding $9 \%$ $(\mathrm{v} / \mathrm{v})$ of $\mathrm{HCl}[1 \mathrm{~N}]$ in the solution. The mix was then immersed in a water bath at $60{ }^{\circ} \mathrm{C}$ for $120 \mathrm{~h}$, until the formation of suitably long amyloid fibrils, as verified by AFM. No purification of the amyloid fibers was performed. The solution was then diluted ten times before depositing a $10 \mu \mathrm{l}$ drop over the superhydrophobic substrate with a hypodermic syringe microneedle. The drop was, thereafter, dried at room temperature and 50\% humidity for 3 hours.

\section{Sample characterization by AFM and SEM}

The formation of amyloid fibrils at least $12 \mu \mathrm{m}$ long (since this is the exact distance between the pillars edge in radial direction) was monitored by AFM. A $30 \mu \mathrm{l}$ drop was withdrawn every 12 hours and spotted on a freshly cleaved mica sheet. It was let adsorb for 10 minutes and then rinsed thoroughly with MilliQ water. Finally it was $\mathrm{N}_{2}$ dried and ready for measurement. JPK Nanowizard III mounted on inverted Olympus IX73 microscope was used for the measurement at relative humidity below 15\%. XSC11 AFM probes (MikroMasch, Nanoworld AG) with nominal resonance frequency of about $150 \mathrm{kHz}$ and nominal force constant of $7 \mathrm{~N} / \mathrm{m}$ were run in tapping mode for the topography measurement.

2 After drop-casting the solution over the pillars top, we let it dry for 3 hours, then the deposition of the fibers was verified by a Quanta 200 FEG Scanning Electron Microscope (FEI) at $5 \mathrm{kV}$ beam voltage and 
11464 pA current. Before imaging, the sample was sputter coated with $2 \mathrm{~nm}$ of Iridium, to avoid charging 115 effects.

117 4. Raman spectroscopy of lysozyme amyloid fibrils

Laser micro-Raman spectroscopy was performed in confocal back scattering geometry by exciting the sample with $532 \mathrm{~nm}$ linearly polarized laser wavelength (Coherent Compass Sapphire Laser, $75 \mathrm{~mW}$ ) at 4 mW power on a WiTec Raman spectrometer (Alpha300 RA) with Andor CCD detector (DU970N) cooled 121 at $-65^{\circ} \mathrm{C}$ and a $100 \mathrm{x}$ objective (Zeiss, EC EPIPLAN NEOFLUAR, 0.9 NA). Measurements of the

122 lysozyme powder dissolved in MilliQ water and lysozyme fibrils in acidic solution were performed after 123 depositing the sample on a $\mathrm{CaF}_{2}$ substrate to minimize signal background. The range of pillars height and 124 spacing useful for effective deposition is chosen to be bigger than the focal length and lateral dimension 125 of the focus of the objective. In this way the excitation volume is optimized to minimize the background 126 from the silicon substrate. For the measurement of the fiber anisotropy, the sample was oriented at $0^{\circ}$ 127 (parallel) or $90^{\circ}$ (perpendicular) with respect to the polarization of the excitation laser. Data analysis was 128 carried out as follows: at least ten measurements per sample were acquired, baseline subtracted in the 129 range 1100-1800 $\mathrm{cm}^{-1}$ (by grade 5 polynomial fitting) and averaged. Afterwards all the spectra were 130 normalized to the Raman peak intensity at $1450 \mathrm{~cm}^{-1}$ to compare data. Amide I spectral region was fitted 131 to a band described by a mixture of Lorentzian and Gaussian functions.

\section{Results and Discussion}

\section{Amyloid fibers on SHS}


molecules, free from the presence of a substrate, clean and not contaminated by debris, and highly

137 oriented along one given direction. This effect is obtained by the shear flow generated in the receding

138 meniscus of the drop which contains the reaction solution (including fibrils, oligomers and native

139 enzyme) and is evaporating over the micro-patterned SHS, and it was first demonstrated by De Angelis et

140 al. [1]. In this work, cylindrical micro-pillars arranged in a circular pattern were used. Pillars inter-

141 distance was $12 \mu \mathrm{m}$. The result of the dehydration process can be appreciated in Fig. 1: in Fig. 1a, four

142 different drying areas emerge as a result of the evaporation of the drop maintained in quasi-spherical

143 configuration by the SHS. The arrows indicate the drying direction, radial with respect to the center of the

144 drop. Along the drying pattern, an area with salt residues is evident on the right bottom corner, preceding

145 the area where the fibers are effectively extended hanging from the pillars edge (green line); this is then

146 followed by a zone with a larger and amorphous residue on the pillars top (starting at the red line)

147 followed by the edge of the collapsed drop (delimited by the blue line), where most of salt and fibers

148 monomers, not long enough to extend across the pillars, are accumulated. This qualitative result partially

149 supports the hypothesis that the SHS has a sieving effect on the drying solution. Fig. 1b-c are a sequence

150 of zoom-in pictures better elucidating the formation of the fiber bundle hanging out from the top of a

151 pillar in the part of Fig. 1a colored in green. The image of pillar top shows a darker area, where it is

152 clearly visible the semicircular drying periphery of the solution bearing radial direction, indicated by the

153 red arrows (Fig. 1b). A bundle of fibers is then formed, by coalescence of the fibers aided by the

154 centripetal drying process (Fig. 1c). Following this organization process the fibers align over the

155 interested area bridging consecutive pillars at a pillar-to-pillar distance of $12 \mu \mathrm{m}$. In the case shown in

156 Fig. 2c, the generated fiber was quite thick, while in other cases thinner fibers were extended. For

157 instance, in Fig. 2a, the fibers pinned at the edge of the pillar top have diameters of about $11 \mathrm{~nm}$. This

158 value includes the $2 \mathrm{~nm}$ of Iridium coating, such that the actual thickness is slightly reduced. The

159 population distribution is then quite wide with fibers measuring also several tens of nanometers, mainly 
depending on the distance from the center, such that a concentration effect is evident (the closer to the 161 drop center, the more concentrated the solution, the thicker the fiber).

Amyloid fibrils can aggregate in different fashion to the limit, however, of the minimum size of the single amyloid fibril (called protofibril) at about $3 \mathrm{~nm}$ in diameter [22]. This effect of aggregation of multiple fibrils due to concentration can be better explained after considering the metrology of the single fibers as measured by the AFM. Fig. 2b is the AFM measurement topography performed at $120 \mathrm{~h}$ solution incubation time, after a drop of the mixture was spotted and dried over a freshly cleaved mica sheet: amyloid fibrils dimensions are $18 \pm 3 \mathrm{~nm}$ of width, $3.4 \pm 0.6 \mathrm{~nm}$ of height and a length of $19 \pm 7 \mu \mathrm{m}$. The width originates actually from the profile measurement convolved with the tip shape, because it was not possible to deconvolve reasonably the signal. Thus, the measured height was taken into consideration.

170 However it has to be pointed out that slight deformation of the fiber might depend on its adhesion to the 171 mica surface, on the shrinking of the molecule due to dehydration and on the effect of the probe tip 172 applying a force while performing the topography measurement. Moreover, the amyloid fibers have 173 different shapes themselves, where the protofibril can organize into fibrils having from cylindrical to 174 ribbon shape. Because the metrology of the amyloid fiber is beyond the scope of this work, we rely on the 175 literature that reports an average radius of the amyloid protofibril, rather common among the different 176 originating proteins, of about $3 \mathrm{~nm}$, and we assume that the height of the fiber might be similar to this 177 value. Given these premises, we can thus state that the height average measured by the AFM on the fibrils 178 population is in agreement with the literature [23-25]. Amyloid fibers are composed of multiple 179 protofilaments that are intertwined together into a twisted ultrastructure with regular periodicity. In these 180 AFM measurements, two populations were found bearing each a twisting pitch of $76.5 \pm 1.2$ and $96.2 \pm$ $181 \quad 1.7 \mathrm{~nm}$. In conclusion, by comparing the AFM and the SEM measurement, we can argue that the thinnest 182 fibers extended on the pillars top could likely be 2 to 4 protofibrils intertwined together, given the SEM 183 diameter estimation. 


\section{Raman measurement of amyloid fibers on SHS}

Lysozyme is an enzyme protein with antibacterial role and is commonly found in human secretions, macrophage and egg white. Due to its immunity importance and availability, lysozyme 3D structure and sequence have been among the first to be resolved. It has also been a model molecule in the early ages of Raman spectroscopy applied to biomedicine due to the presence of the main aminoacids in its sequence and of the S-S bond. Some major peaks characterize the laser Raman spectroscopy spectrum of a protein in the visible excitation range, related to specific aminoacids (e.g. Phenylanin $\sim 1003 \mathrm{~cm}^{-1}$; Tyrosine $~ 830$, $850 \mathrm{~cm}^{-1}$; Tryptophan $\sim 763,980,1556 \mathrm{~cm}^{-1}$ ), to specific covalent bonds (S-S $\sim 510 \mathrm{~cm}^{-1}$; C-H (def.) $\sim 1450$ $\mathrm{cm}^{-1}$ and C-N $\sim 1130 \mathrm{~cm}^{-1}$ ) and to secondary structure (Amide III $\sim 1240 \mathrm{~cm}^{-1}$ and Amide I $\sim 1650 \mathrm{~cm}^{-1}$ ) [15]. Our interest particularly focuses on the secondary structure fingerprints, given that a globular protein, such as HEWL, will change its three dimensional structure by re-organizing the population distribution of its secondary structures in the conversion to amyloid protein. The Amide I band (Raman shift: 1620-1690 $\mathrm{cm}^{-1}$ ), mainly due to the $\mathrm{C}=\mathrm{O}$ stretching of the peptide carbonyl group, will be used in this work to describe the protein secondary structure in the visible excitation range. After the discovery that every protein can turn into amyloid fibril, lysozyme was readily used to study the onset of these pathological aggregates. The lysozyme powder is processed to obtain the amyloid fibrils, usually in denaturing conditions and high temperature. The result is the destabilization of the protein tertiary structure into an open globular feature which then rearranges into sheets of $\beta$-strands, since this type of aggregation is energetically favored [26]. The structure of these assemblies has been somewhat controversial; however, a general pattern common too many proteins is nowadays accepted, where the core of the amyloid fiber is made up of $\beta$-sheets running perpendicular to the fiber and packed together by side-chains interactions [27]. Aside from this core, other secondary structures such as $\alpha$-helices, random coils, loops, and other unordered structures are detected, depending on the sequence of the protein. Besides, proteins showing the tendency to form amyloid fibrils always possess a susceptible aminoacid 
sequence which is prone to alteration $[28,29]$. It has to be pointed out that different preparation conditions can give rise to amyloid polymorphs originating from the same protein, such that slight

210 variability exists in the fibrils structure and related analysis outcomes. All of these characteristic 211 secondary structures have been assigned specific Raman shifts in the Amide I band of the Raman 212 spectrum. As a rule of thumb, the Amide I band will be mostly populated by the $\beta$-sheet assigned peak, 213 thus having a center at $1672 \mathrm{~cm}^{-1}[16,17,30]$. In this work, by suspending bundles made of few fibrils 214 between pillars, we expect a purified Raman signal unmistakably elucidating the secondary structure 215 typical of the amyloid molecule.

In Fig. 3 the Raman spectra in the range $1500-1800 \mathrm{~cm}^{-1}$ of the lysozyme in MilliQ water (Lys-MQ), 217 the lysozyme in the acidic solution after $120 \mathrm{~h}$ of incubation in hot bath (Lys-HCl) and the lysozyme fiber 218 bridged across pillars (Fibril_Pill) are plotted. Focusing on the Amide I band in the range 1620-1690 $\mathrm{cm}^{-1}$, 219 an upshift of the Amide I peak is readily recognizable when moving from globular protein to amyloid 220 fiber, where the peak apex moves from $1662 \mathrm{~cm}^{-1}$ to $1672 \mathrm{~cm}^{-1}$. In particular, the curve Lys-MQ has a 221 peak at $1662 \mathrm{~cm}^{-1}$; the Lys-HCl has a peak centered at $1667 \mathrm{~cm}^{-1}$; the Fibril_Pill bears a remarkably 222 narrow peak at $1672 \mathrm{~cm}^{-1}$. This shift is already assigned in the literature to the transition from globular 223 protein to amyloid fibril, where the peak at $1672 \mathrm{~cm}^{-1}$, might be assigned to the $\beta$-sheet predominant 224 component of the Amide I region [17]. The measurement in which the laser polarization is parallel to the 225 fiber is giving the better results in terms of the intensity of the peak attributed to the $\beta$-sheet secondary 226 structure, as compared to the spectrum obtained when the fiber is oriented perpendicularly to it: in fact, 227 the peak intensity increases nearly three-folds (Fig. 4). This result shows that the $\beta$-sheets are 228 preferentially oriented perpendicular to the fiber axis, as previously demonstrated by Sereda et al. [19], in 229 a polarized Raman study performed on insulin amyloid fibers aligned by the coffee ring stain effect. 230 However in their work, there was a need for previous purification of the fibers and the analysis was not 231 restricted to few molecules. Here we obtained the similar enhanced effect on the Raman spectrum, but 
with the great advantage to provide with our method single bundles composed of few molecules that,

233 while purified, are oriented according to the patterned surface. In fact, the drop-casted solution is 234 populated by several species (globular enzymes, amyloid seeds, etc.), as it is shown in the AFM 235 topography image in Fig. 2b. These species are largely removed from the extended fibers after the drop 236 dries over the pillars, thanks to the sieving effect of the drying process on the SHS.

A deeper analysis of the results obtained from Raman spectroscopy on oriented fibers and electron microscopy characterization of these samples is beyond the scope of this publication and will be reported elsewhere in more detail.

\section{Conclusions}

In this work we have demonstrated a method to generate suspended amyloid fibrils highly oriented in a pre-defined direction by the use of a SHS. This method provides fibrils free of any 243 contaminant and residual, decoupled from any supporting substrate, ready for further Raman or electron 244 microscopy analysis. The sample is obtained by the shear flow generated at the rim of a drop, casted over 245 a micro-patterned SHS. The receding meniscus combined with the shear flow and the pinning of the 246 fibrils on pillars edge is responsible for the alignment and purification of the amyloid fibrils. In particular, 247 we were able to suspend single/few amyloid fibrils of different diameters down to about $11 \mathrm{~nm}$, as 248 verified by the SEM imaging. The micro-Raman analysis of the sample, as expected, revealed a 249 progressive upshift of the Amide I band main peak (from 1662 to 1667 to $1672 \mathrm{~cm}^{-1}$ ) when the transition 250 from pure-lysozyme to lysozyme-amyloid-fibrils solution (mix of fibers and oligomers) and to lysozyme 251 amyloid fibrils suspended on pillars was considered. This testifies for the increased contribution of the $\beta$ 252 sheet secondary structure typical in amyloid fibrils. The distinctive anisotropic property of the amyloid 253 fiber was also revealed with an enhanced Raman signal peaked at $1672 \mathrm{~cm}^{-1}$ after orienting the stretched 254 fibrils parallel to the polarization of the incident laser. In conclusion, we would remark that this method 
could be considered as well suited to characterize any type of anisotropic fiber crystals. We foresee the extension of the method for studying other protein molecules not only by Raman spectroscopy but also by transmission electron microscopies, both in diffraction and direct imaging configuration and x-ray crystallography. In fact, a single preparation technique, such as the dehydration on SHS device, provides a sample available for characterization under three complementary techniques, simplifying and reducing processing times. Future and alternative applications include, but are not limited to, the generation of patterned surfaces when bio-compatible material is desirable, thanks not only to the availability of the

262 protein molecule (and eventual new synthesis of simpler sequences), but also to the possibility of 263 chemical-biological modification of the starting material.

\section{Acknowledgements}

265 The authors acknowledge financial support from the KAUST start-up funding and from Italian Ministry 266 of Health under the projects: Project no.: GR-2010-2320665 and Project no.: GR-2010-2311677.

\section{References}

268 [1] F. De Angelis, F. Gentile, F. Mecarini, G. Das, M. Moretti, P. Candeloro, M.L. Coluccio, G. Cojoc, A. 269 Accardo, C. Liberale, R.P. Zaccaria, G. Perozziello, L. Tirinato, A. Toma, G. Cuda, R. Cingolani, E. Di 270 Fabrizio, Breaking the diffusion limit with super-hydrophobic delivery of molecules to plasmonic 271 nanofocusing SERS structures, Nat Photon 5(11) (2011) 682-687.

272 [2] F. Gentile, M. Moretti, T. Limongi, A. Falqui, G. Bertoni, A. Scarpellini, S. Santoriello, L. 273 Maragliano, R. Proietti Zaccaria, E. di Fabrizio, Direct Imaging of DNA Fibers: The Visage of Double 274 Helix, Nano letters 12(12) (2012) 6453-6458.

275 [3] F. Gentile, G. Das, M.L. Coluccio, F. Mecarini, A. Accardo, L. Tirinato, R. Tallerico, G. Cojoc, C. 276 Liberale, P. Candeloro, P. Decuzzi, F. De Angelis, E. Di Fabrizio, Ultra low concentrated molecular 
detection using super hydrophobic surface based biophotonic devices, Microelectron Eng 87(5-8) (2010) 798-801.

[4] F. Gentile, M.L. Coluccio, N. Coppedè, F. Mecarini, G. Das, C. Liberale, L. Tirinato, M. Leoncini, G. Perozziello, P. Candeloro, F. De Angelis, E. Di Fabrizio, Superhydrophobic Surfaces as Smart Platforms for the Analysis of Diluted Biological Solutions, ACS Applied Materials \& Interfaces 4(6) (2012) 32133224.

[5] T. Limongi, F. Cesca, F. Gentile, R. Marotta, R. Ruffilli, A. Barberis, M. Dal Maschio, E.M. Petrini, S. Santoriello, F. Benfenati, E. Di Fabrizio, Nanostructured Superhydrophobic Substrates Trigger the Development of 3D Neuronal Networks, Small 9(3) (2013) 402-412.

[6] D. Bensimon, A.J. Simon, V. Croquette, A. Bensimon, Stretching DNA with a Receding Meniscus: Experiments and Models, Physical Review Letters 74(23) (1995) 4754-4757.

[7] M. Marini, A. Falqui, M. Moretti, T. Limongi, M. Allione, A. Genovese, S. Lopatin, L. Tirinato, G. Das, B. Torre, A. Giugni, F. Gentile, P. Candeloro, E. Di Fabrizio, The structure of DNA by direct imaging, Science Advances 1(7) (2015).

[8] M. Marini, G. Das, R. La Rocca, F. Gentile, T. Limongi, S. Santoriello, A. Scarpellini, E. Di Fabrizio, Raman spectroscopy for detection of stretched DNAs on superhydrophobic surfaces, Microelectron Eng 119 (2014) 151-154.

[9] M. Marini, T. Limongi, A. Falqui, A. Genovese, M. Allione, M. Moretti, S. Lopatin, L. Tirinato, G.

Das, B. Torre, A. Giugni, F. Cesca, F. Benfenati, E. Di Fabrizio, Imaging and structural studies of DNAprotein complexes and membrane ion channels, Nanoscale (2017).

[10] M. Marini, M. Allione, B. Torre, M. Moretti, T. Limongi, L. Tirinato, A. Giugni, G. Das, E. di Fabrizio, Raman on suspended DNA: Novel super-hydrophobic approach for structural studies, Microelectron Eng 175 (2016) 38-42.

[11] F. Chiti, C.M. Dobson, Protein misfolding, functional amyloid, and human disease, Annu Rev Biochem 75 (2006) 333-66. 
[12] C.M. Dobson, Protein folding and misfolding, Nature 426(6968) (2003) 884-90.

[13] M. Egli, Diffraction Techniques in Structural Biology: Overview for unit 7 "Biophysical Analysis of Nucleic Acids” in: Current Protocols in Nucleic Acid Chemistry, Current protocols in nucleic acid chemistry / edited by Serge L. Beaucage ... [et al.] CHAPTER 7 (2010) Unit-7.13.

[14] R. Tuma, Raman spectroscopy of proteins: from peptides to large assemblies, J Raman Spectrosc 36(4) (2005) 307-319.

[15] A. Rygula, K. Majzner, K.M. Marzec, A. Kaczor, M. Pilarczyk, M. Baranska, Raman spectroscopy of proteins: a review, J Raman Spectrosc 44(8) (2013) 1061-1076. [16] K. Huang, N.C. Maiti, N.B. Phillips, P.R. Carey, M.A. Weiss, Structure-specific effects of protein topology on cross-beta assembly: studies of insulin fibrillation, Biochemistry 45(34) (2006) 10278-93.

312 [17] N.C. Maiti, M.M. Apetri, M.G. Zagorski, P.R. Carey, V.E. Anderson, Raman spectroscopic 313 characterization of secondary structure in natively unfolded proteins: alpha-synuclein, Journal of the 314 American Chemical Society 126(8) (2004) 2399-2408.

315 [18] T. Lefevre, M.E. Rousseau, M. Pezolet, Protein secondary structure and orientation in silk as 316 revealed by Raman spectromicroscopy, Biophys J 92(8) (2007) 2885-95.

317 [19] V. Sereda, I.K. Lednev, Polarized Raman Spectroscopy of Aligned Insulin Fibrils, J Raman 318 Spectrosc 45(8) (2014) 665-671.

319 [20] V. Sereda, M.R. Sawaya, I.K. Lednev, Structural Organization of Insulin Fibrils Based on Polarized 320 Raman Spectroscopy: Evaluation of Existing Models, J Am Chem Soc 137(35) (2015) 11312-20.

321 [21] M.R.H. Krebs, D.K. Wilkins, E.W. Chung, M.C. Pitkeathly, A.K. Chamberlain, J. Zurdo, C.V. 322 Robinson, C.M. Dobson, Formation and seeding of amyloid fibrils from wild-type hen lysozyme and a 323 peptide fragment from the beta-domain, Journal of Molecular Biology 300(3) (2000) 541-549.

324 [22] L.C. Serpell, M. Sunde, M.D. Benson, G.A. Tennent, M.B. Pepys, P.E. Fraser, The protofilament 325 substructure of amyloid fibrils, Journal of Molecular Biology 300(5) (2000) 1033-1039. 

protofilament structure of insulin amyloid fibrils, Proceedings of the National Academy of Sciences 99(14) (2002) 9196-9201.

[24] A.K. Chamberlain, C.E. MacPhee, J. Zurdo, L.A. Morozova-Roche, H.A.O. Hill, C.M. Dobson, J.J. Davis, Ultrastructural Organization of Amyloid Fibrils byAtomic Force Microscopy, Biophys J 79(6)

331 (2000) 3282-3293.

332 [25] M. Moretti, C. Canale, M. Francardi, S. Dante, F. De Angelis, E. Di Fabrizio, AFM characterization 333 of biomolecules in physiological environment by an advanced nanofabricated probe, Microsc Res 334 Techniq 75(12) (2012) 1723-1731.

335 [26] D.R. Booth, M. Sunde, V. Bellotti, C.V. Robinson, W.L. Hutchinson, P.E. Fraser, P.N. Hawkins, 336 C.M. Dobson, S.E. Radford, C.C. Blake, M.B. Pepys, Instability, unfolding and aggregation of human 337 lysozyme variants underlying amyloid fibrillogenesis, Nature 385(6619) (1997) 787-93.

338 [27] T.R. Jahn, O.S. Makin, K.L. Morris, K.E. Marshall, P. Tian, P. Sikorski, L.C. Serpell, The common 339 architecture of cross-beta amyloid, J Mol Biol 395(4) (2010) 717-27.

340 [28] A.P. Pawar, K.F. Dubay, J. Zurdo, F. Chiti, M. Vendruscolo, C.M. Dobson, Prediction of

341 "aggregation-prone" and "aggregation-susceptible" regions in proteins associated with neurodegenerative 342 diseases, J Mol Biol 350(2) (2005) 379-92.

343 [29] A. Lakshmanan, D.W. Cheong, A. Accardo, E. Di Fabrizio, C. Riekel, C.A.E. Hauser, Aliphatic 344 peptides show similar self-assembly to amyloid core sequences, challenging the importance of aromatic 345 interactions in amyloidosis, Proceedings of the National Academy of Sciences 110(2) (2013) 519-524. 346 [30] D. Kurouski, R.P. Van Duyne, I.K. Lednev, Exploring the structure and formation mechanism of 347 amyloid fibrils by Raman spectroscopy: a review, The Analyst 140(15) (2015) 4967-80. 

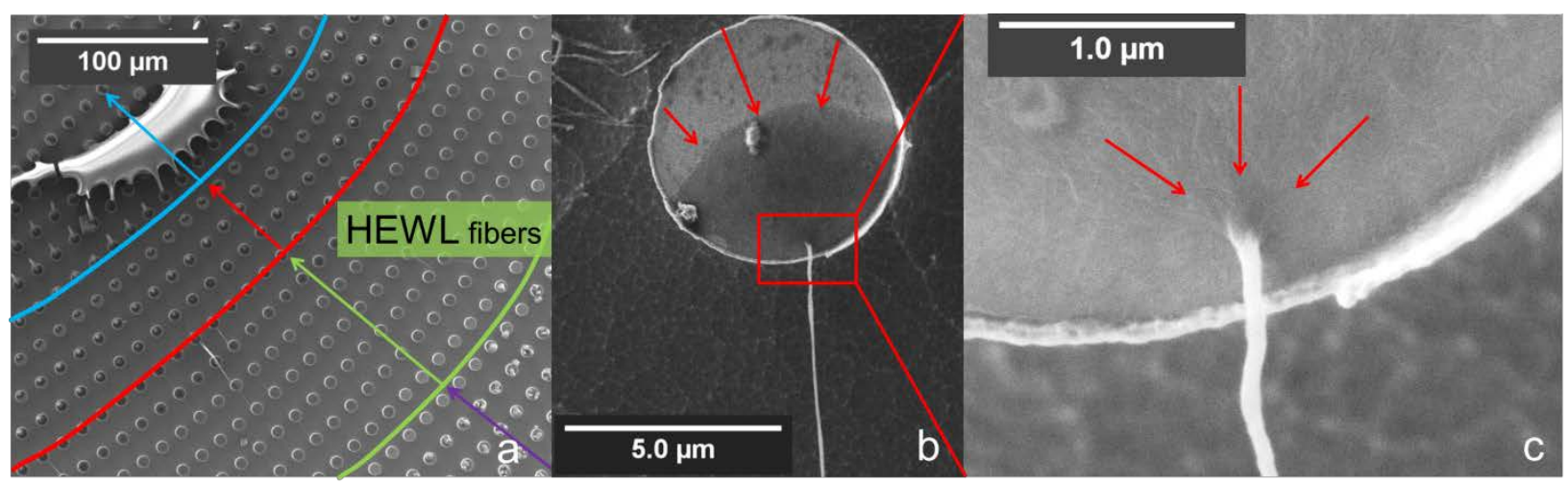

Fig. 1: SEM micrographs with cartoons elucidating the drying mechanism of the drop solution

353 spotted over the SHS. (a) A view over a large area which includes the side of the evaporated drop (top

354 left) and several drying areas evidenced by different marker colors. The green tag indicates the area were

355 the HEWL fibers are stretched over the pillars' top. The arrows indicate the drying direction; (b) a pillar

356 top with red arrows evidencing the drying shape and direction; (c) the area in the red square in (b) imaged

357 at higher magnification to show the coalescence of the fibers to one point (indicated by the red arrows)

358 where they are pinned out of the pillar top. The images were contrast and brightness corrected for better 359 visualization of the amyloid fibers. 


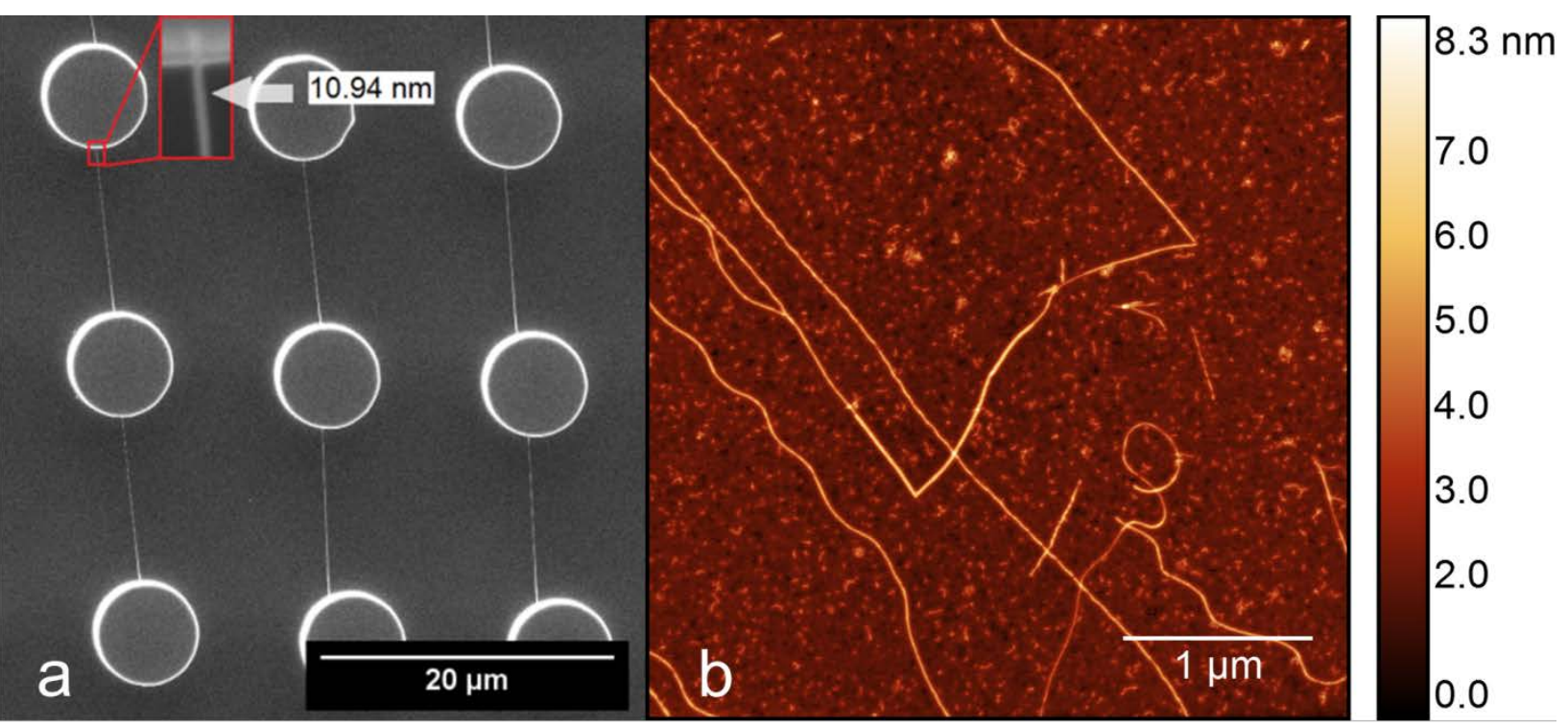

Fig. 2: (a) SEM micrograph of the suspended fibers over super-hydrophobic pillars. Minimum

362 diameter measured was about $11 \mathrm{~nm}$ (inset). (b) AFM topography of lysozyme amyloid fibrils deposited 363 on mica sheet after 120 hours of incubation. The height of the fibrils is about $3 \mathrm{~nm}$. 


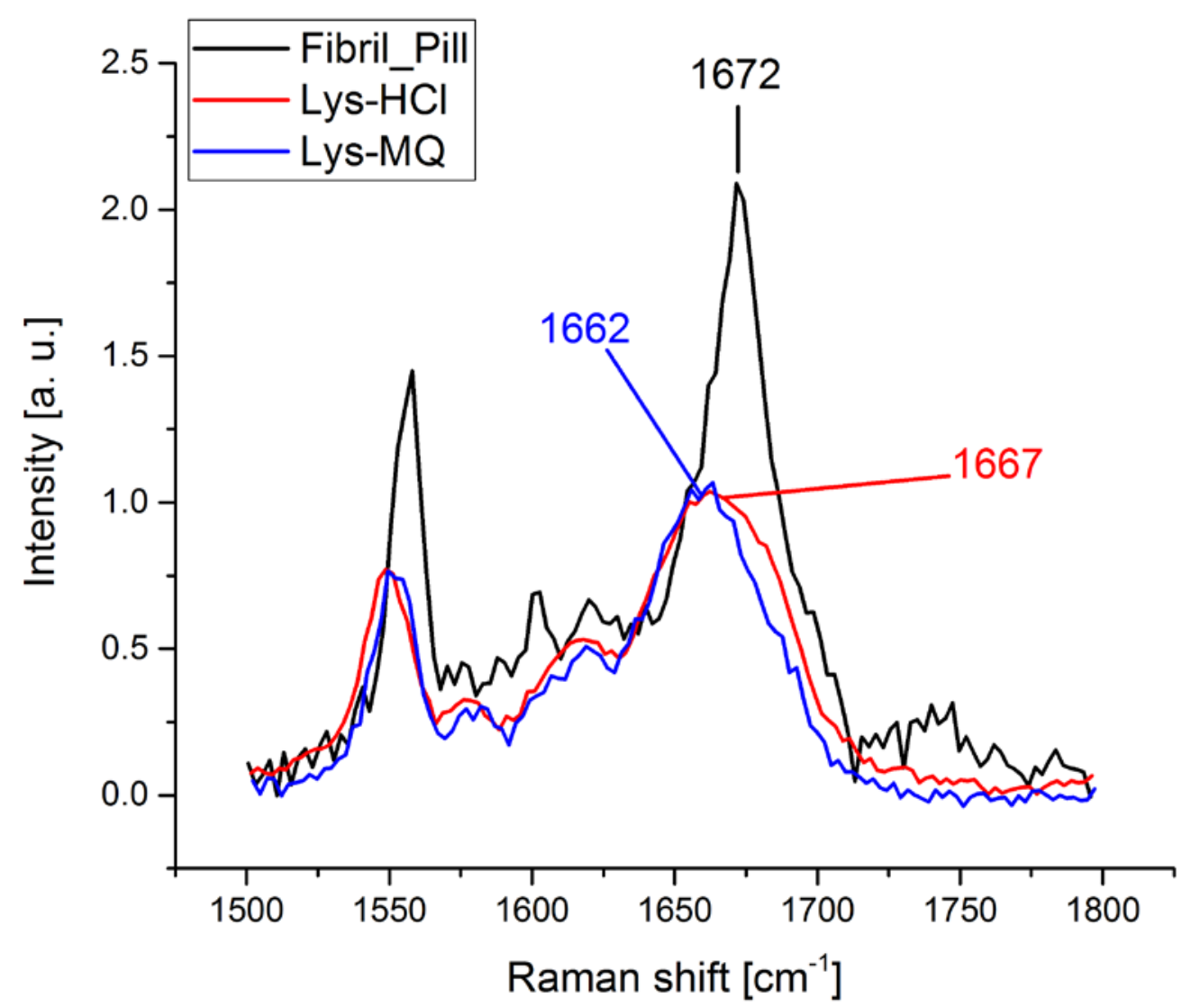

364

Fig. 3: Raman spectrum and peak assignment of the Amide I region for the HEWL in MilliQ water,

366 named Lys-MQ, in blue, HEWL in acidic solution, named Lys-HCl, in red and the HEWL fibers bridging

367 the pillars top, named Fibril_Pill, in black. Raman spectra were normalized to the $1450 \mathrm{~cm}^{-1}$ peak. The 368 assigned Raman peak was obtained by fitting Voigt function to a single peak in the Amide I band. 


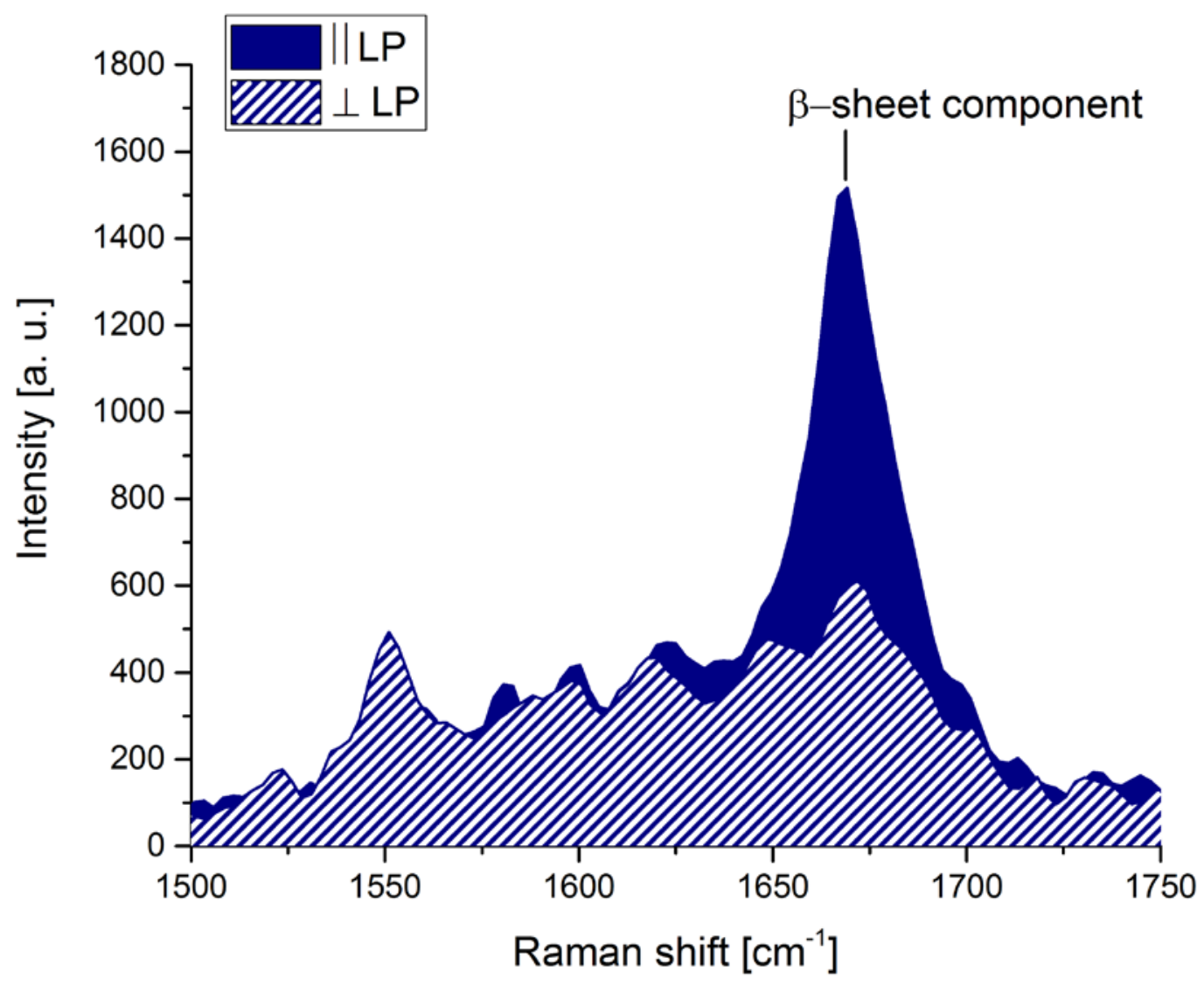

$370 \quad$ Fig. 4: Raman spectra of the amyloid fibers extended over the SHS with parallel (\|LP, filled blue

371 spectrum) and perpendicular ( $\perp \mathrm{LP}$, striped blue spectrum) orientation with respect to the exciting laser

372 polarization (LP) in the region $1500-1800 \mathrm{~cm}^{-1}$ : the peak of the $\beta$-sheet component at $1672 \mathrm{~cm}^{-1}$ is

373 remarkably enhanced in the case of the laser polarization parallel to the fiber direction. 